\title{
La gestión local Eslabón perdido de las políticas sociales orientadas al desarrollo local
}

\section{Magdalena Chiara*}

La gestión entró en la agenda como "cuestión" de modo diverso en el transcurso de la última década: desde el inicial diagnóstico acerca de la ineficiencia del aparato estatal que dio lugar a los distintos procesos de reforma, pasando por la batería instrumental del gerenciamiento de programas y la necesidad de fortalecimiento de la sociedad civil.

La profundidad de la crisis y los desafíos de la política social nos plantean la pregunta acerca de las condiciones bajo las cuales puede contribuirse al desarrollo de formas orientadas a la economía social y el desarrollo local y el papel que en el desarrollo de estas condiciones tiene la tarea de generar espacios locales de gestión pública.

* Antropóloga, investigadora y consultora experta en gestión de políticas sociales. Desde mediados de la década del 80 ha trabajado en investigación y gestión en problemas urbanos en distintos campos. Desde 1995 es investigadora docente del Instituto del Conurbano de la UNGS. Ha publicado diversos artículos en revistas especializadas y capítulos de libros referidos a las políticas sociales urbanas. Ha sido funcionaria municipal y provincial y consultora de organismos públicos e internacionales en gestión y evaluación de políticas sociales. Ha sido docente en la Universidad de Buenos Aires y del Instituto Nacional de la Administración Pública. Desde el 2002 es Directora del Instituto del Conurbano de la UNGS. E-mail: magdachiara@infovia.com.br 
La complejidad y diversidad que presentan las experiencias nacionales hablan de la necesidad mirar la práctica aportando conceptos que nos permitan reflexionar críticamente sobre ella, pasando en limpio aprendizajes que puedan recuperarse en acciones públicas desde distintos espacios.

Este trabajo propone pensar a la gestión pública desde los actores, su complejidad inherente y relacional y plantear algunas coordenadas conceptuales de un debate que consideramos sustantivo (no instrumental) en el desarrollo politicas sociales promotoras de formas de producción y reproducción social basadas en las necesidades de las comunidades.

\section{A procesos complejos, entramados complejos: ¿quienes son los actores del desarrollo local?}

Para avanzar en la comprensión de estas cuestiones, es necesario definir a qué nos referimos cuando hablamos de desarrollo local y por qué consideramos que estos procesos pueden ser promovidos en distintas escalas.

Con desarrollo local estamos aludiendo a procesos complejos a través de los cuales - por medio de diferentes estrategias - se aumentan y desarrollan las capacidades de los actores de modo de orientarlos a la mejora integral de la calidad de vida de la población en sus aspectos económicos (ingreso, satisfacción de necesidades, trabajo), culturales (identidad), sociales (integración social, oportunidades de acceso a salud, educación) y políticos (participación en las decisiones públicas, legitimidad de los representantes, entre otros). ${ }^{1}$

Lo local - desde esta perspectiva - no está apelando necesariamente a una escala micro (barrial o vecinal) sino a aquella que se constituye en un territorio configurado por la historia de relación entre los actores y por las cuestiones que los ponen en relación (Coraggio, 2003).

Esto significa que un actor se constituye en actor de lo local en la medida en que actúa de manera comprometida con relación a cuestiones socialmente problematizadas en la escena local. En ese sentido, consideramos como "actores" de procesos de desarrollo local al gobierno local, a organismos

1 Desde esta perspectiva, la UNGS viene desarrollando desde el año 2001 tres ediciones del Curso de Posgrado en Desarrollo Local para Áreas Metropolitanas e iniciará en agosto del 2004 la Carrera de Especialización en Desarrollo Local. 
provinciales y nacionales, a una ONG de desarrollo de alcance regional o provincial, a organizaciones sociales de base, sindicatos, todos ellos preocupados, comprometidos y actuando en relación con esos procesos locales. Desde esta perspectiva es la categoría de "acción orientada al desarrollo local" la que define la inclusión como actor en esos procesos.

Enfatizamos entonces que esto no significa que todos los actores por el solo hecho de actuar en la escena local sean relevantes para los procesos de desarrollo en esa localidad; dicho de otro modo, que su relevancia pueda ser definida por fuera del sentido de su acción. En esta perspectiva, la definición que propone J. Arocena permite captar la diversidad pero a la vez el sentido de la acción cuando afirma, "bajo la fórmula de actor local, entendemos a todos aquellos agentes que en el campo político, económico, social y cultural, son portadores de propuestas que tienden a capitalizar mejor las potencialidades locales" (Arocena, 1988). El corte aquí es definido en función del sentido, la intencionalidad y los propósitos de la acción.

La acción pública de los actores locales así definidos tiene alcances diferenciales conforme sean las cuestiones desde las cuales ésta es interpelada. Esto significa - en términos concretos - que su alcance territorial variará según los marcos de la experiencia, la problemática y las cuestiones sobre las cuales pongan éstos en juego su capacidad de acción pública (barrio, localidad, cuenca, región sanitaria, municipio, distrito escolar o región educativa, provincia, nación). La complejidad se torna aún mayor en tanto estos procesos nos enfrentan con actores (individuos, grupos o instituciones) cuya actuación pública no sólo tiene - como hemos dicho - un alcance territorial diferente, sino que también sus intereses se despliegan en más de uno de esos territorios, con influencias más o menos explícitas sobre el ámbito local.

Complejizando aún más el fenómeno del cual estamos intentando dar cuenta, conforme la dimensión en la cual legitiman su acción, estos actores están ligados con distintos aspectos de los procesos de desarrollo; las decisiones (político - institucionales), las técnicas particulares (expertos - profesionales) y la acción sobre el terreno ${ }^{2}$ son - en términos muy generales - las tres grandes dimensiones en que se puede desplegar la acción; esto fortalece la idea de "territorio" como resultado de una compleja construcción.

2 Recuperamos aquí la tipología propuesta por F. Barreiro Cavestany (1988). 
En este sentido entonces, la definición misma del territorio (como alcance y a la vez escena de su acción) es resultado un proceso de construcción social, político y también simbólico. Los procesos de concertación comprendidos en la gestión pública van construyendo política y también simbólicamente una territorialidad de referencia en la relación entre los distintos actores y en las cuestiones que ingresan a la agenda local.

La construcción de un territorio de referencia así definido se convierte en "tarea" cuando se inician estos procesos de trabajo colectivo y no puede ser pensada como pre-requisito de la acción para lograr el desarrollo local, ni tampoco como una decisión exclusivamente técnica. Las distintas experiencias nacionales y latinoamericanas relativas a procesos de planeamiento participativo y estratégico local ponen en evidencia ese trabajoso proceso de construcción colectiva.

Si centramos el análisis en los actores, la reflexión debe poner en el centro al sistema de relaciones que éstos generan, que se va modificando a lo largo del tiempo y que, al mismo tiempo, los condiciona. ${ }^{3}$ Esto se funda en el supuesto que los actores no definen aisladamente su acción pública, no tienen una homogénea capacidad de acción en el ámbito público, ni tampoco permanecen constantes a lo largo del tiempo. ${ }^{4}$ Indagar en la capacidad de actuación de los actores en el marco del sistema de relaciones que los constituye y condiciona, implica poner en el centro de la reflexión el desarrollo local en tanto todas las categorías comprendidas en ese campo ("desarrollo", "sociedad", "políticas", "identidad", "cultura", "sistema político", "participación”) suponen la existencia de actores que tienen capacidad de actuar en lo local (Arocena, 2003, p. 2).

Estos actores y las relaciones que establecen entre sí constituyen un entramado de intereses, con dinámicas de relación y capacidades de acción pública diferenciales. Es en el marco del "entramado" local más que en las relaciones institucionales formales (aunque ellas condicionan su estructura

3 Remito aquí a la conferencia inaugural "La tensión actor-sistema en los procesos contemporáneos de desarrollo" realizada por Jose Arocena en la Escuela Regional de Verano América Latina y el Caribe (2003).

4 Para esta perspectiva de análisis aplicada a la gestión local de los programas con financiamiento externo ver Chiara, M y Di Virgilio, M. M. (2003). 
y dinámica), que los actores públicos y privados "toman decisiones sobre el uso de recursos comunes con respecto a un determinado problema". ${ }^{5}$ Este entramado de intereses, preexiste a las políticas (condicionando su implementación) y resulta también modificado por ellas.

A fin de dar cuenta de la complejidad de cada entramado, una primera pregunta que debemos hacernos es acerca de quiénes son sus actores. Estos actores son de distinto tipo: gobiernos locales y diferentes áreas del ejecutivo y legislativo municipales, organismos y programas provinciales y nacionales que actúan en la escena local, organizaciones sociales de base, organizaciones específicas como las de tierras y vivienda o salud, ONGs de alcance regional o nacional, sindicatos, escuelas, universidades, los medios de comunicación, entre otros.

El problema que se nos plantea, tanto en el análisis como en la intervención, es que no siempre los individuos, grupos o instituciones (que forman parte de este entramado y están comprometidos con esos procesos) tienen la misma capacidad de actuación. Si bien las capacidades están latentes, su mera existencia no da lugar de manera automática a la actuación pública. La construcción y deconstrucción de los actores está marcada así por el conjunto de las relaciones sociales y políticas en el marco de las cuales esos actores definen su acción y también por las representaciones que se construyen en el marco de dichas relaciones.

En este punto, una política pública nacional y provincial orientada hacia el desarrollo local puede incidir positivamente sobre este espacio que sabemos como configuración particular y única - no sólo desde las reglas de juego (tendiendo a modificar las relaciones sociales y políticas), sino también desde las representaciones (las visiones sobre el proyecto de sociedad, la política, la legitimidad de la representación, la distribución del poder, etc.).

5 Para el concepto de "entramado" ver Jordana (1995) y Subirats, (1989). 


\section{Un mapa de actores modelado por la crisis y las políticas públicas: las "marcas" de la gestión pública local}

Los actores a los que hemos hecho referencia han sufrido una doble transformación. Por una parte, la derivada de los cambios abruptos en las condiciones de reproducción de la vida de los que ellos mismos han sido y son sujetos; la incidencia de la pobreza se duplicó en la mayoría de las provincias entre el año 1998 y 2002, mientras que la indigencia llegó a triplicarse en el mismo periodo. ${ }^{6}$

Estos datos ponen en evidencia transformaciones profundas en las condiciones de reproducción de la vida de estos individuos, grupos y comunidades pero a la vez los aceleradísimos cambios en los problemas y cuestiones con los cuales deben enfrentarse y en función de los cuales re-elaborar representaciones, acordar estrategias y construir prácticas asociadas. La fragmentación y la dificultad para generar estrategias concertadas en estos tres planos (representaciones, estrategias y prácticas) puede ser interpretada también desde la brutalidad de estos cambios.

Por otra parte, los actores han visto modificada en la última década su propia constitución como resultado de los procesos de reforma: los procesos de privatización (en tanto redefinición de las fronteras del Estado con la sociedad y el mercado) y los de descentralización / desconcentración (en tanto replanteo de las tareas hacia dentro del Estado con el consecuente replanteo de las relaciones con la sociedad en los distintos niveles) significaron un profundo replanteo del papel, expectativas y relaciones entre los actores gubernamentales y de la sociedad.

Si nos concentramos particularmente en el papel desarrollado por los gobiernos locales a lo largo del tiempo, vemos que fueron los procesos de

6 Solo para ilustrar la brutalidad de estos cambios, destacamos que la pobreza pasó en el periodo $1998 / 2002$ del $31 \%$ al $62 \%$ en la provincia de Buenos Aires; del $29 \%$ al $58 \%$ en la provincia de Córdoba; del $42 \%$ al $71 \%$ en la provincia de Entre Ríos; $35 \%$ al $62 \%$ en la provincia de Santa Fé; del $6 \%$ al 21\% en la Ciudad de Buenos Aires. En el mismo periodo, la indigencia se triplicó pasando del $9 \%$ al $29 \%$ en la provincia de Buenos Aires; del 7\% al 27\% en la provincia de Córdoba; del 13\% al 39\% en la provincia de Entre Ríos; $11 \%$ al $31 \%$ en la provincia de Santa Fé; del 1\% al 6\% en la Ciudad de Buenos Aires (SIEMPRO, en base a datos EPH, INDEC). 
la década del 90 los que transformaron de manera más decisiva su papel frente a la sociedad. Más allá de las diferencias que podemos encontrar en materia de competencias municipales en los distintos regímenes provinciales, una situación generalizada es el papel relativamente reciente que han tenido los municipios en la gestión de lo social, en lo que algunos autores han dado en llamar la municipalización de la crisis. Y esto ha sido resultado de un cambio que involucró fuertes transformaciones en las políticas sociales, no solo en la descentralización sino también en la privatización (a modo de transferencia de las responsabilidades al mercado y a la familia de la reproducción de la vida de la población) y la focalización (definiendo por la via de canastas básicas de bienes y servicios aquella población que por su vulnerabilidad debía ser atendida por el Estado).

Estos procesos de reforma han tenido consecuencias en los aspectos funcionales de las políticas y también en sus aspectos políticos, es decir, en las cuestiones en torno a las cuales estructuran sus relaciones de poder con la sociedad, poniendo en crisis los tradicionales modelos de representación.

Haciendo particular referencia a la descentralización, los procesos de transferencia tuvieron lugar en un campo de instituciones locales caracterizadas por una baja autonomía y por una importante dependencia en términos de su capacidad de asignación de recursos. Esto dio lugar a la configuración de un nuevo papel para los gobiernos locales frente a la cuestión social, cada vez más acotada a la cuestión de la pobreza, en condiciones de muy baja capacidad de gestión.

Este nuevo papel de los gobiernos locales se fue modelando en el transcurso de la última década en un contexto en el que la agenda nacional y provincial / local fue diferenciándose: mientras el nivel central nacional, fue centrandose en cuestiones macroeconómicas, los niveles provincial local fueron progresivamente ocupándose de las consecuencias de las decisiones tomadas a nivel central en términos de las condiciones de reproducción de la vida. Dada entonces esta brecha entre el nuevo papel y las capacidades de gestión, las consecuencias en clave de crisis de legitimidad y credibilidad de los gobiernos locales cuando apuntaron a ser los "explicadores del ajuste" fueron dramáticas. 
Los procesos de reforma antes aludidos tendieron también a modificar la configuración de las organizaciones sociales comunitarias y las ONGs. Por una parte, la iniciativa de involucrarlas en la gestión de programas contribuyendo con el desarrollo de las capacidades necesarias - jerarquizó su lugar frente a las unidades domésticas como generadoras de intercambios de distinto tipo de recursos y de información de los programas sociales. ${ }^{7}$ En algunos casos, se destaca particularmente la capacidad para articular intersectorialmente recursos en tanto "las redes sociales y las organizaciones comunitarias tienen la particularidad de habilitar el acceso a una multiplicidad de recursos, característica que las diferencia de otras fuentes vinculadas al mercado o al estado" (Di Virgilio, 2003).

Por otra parte, las relaciones construidas en el marco de los programas generaron una dinámica que concluyó en casos muy aislados con el fortalecimiento del entramado local, estableciendo relaciones radiales desde los programas nacionales, exigiendo como prerrequisito la existencia de organizaciones sociales mediadoras, poniendo en competencia a las organizaciones sociales y ONGs por los recursos y, en algunos casos, jerarquizando a las organizaciones sociales frente a los municipios para la gestión de los programas. $^{8}$

El lanzamiento del Plan Jefes y Jefas de HD implicó en cierto sentido una recentralización de las relaciones que se cristalizaron en la década pasada con la reaparición del gobierno nacional como ejecutor del programa más importante de transferencia de ingresos ${ }^{9}$ con las tensiones respecto de la descentralización resultante de las funciones asumidas por las instancias provinciales y el papel previsto en el diseño del programa a los Consejos Consultivos Provinciales y Municipales.

7 Según un estudio realizado en los partidos de J. C. Paz, Malvinas Argentinas, Moreno y San Miguel, el $42 \%$ de los hogares está vinculado con otro a través de intercambios domésticos y un 34\% de los mismos participa en ámbitos de organización comunitaria. Kohan, Fournier (1999).

8 El diseño del Plan Trabajar fue un ejemplo de esto último. Ver Chiara y Di Virgilio (2001).

9 El Plan se desarrolla desde el Ministerio de Trabajo, Empleo y Seguridad Social, tiene prevista una inversión en el 2003 de aproximadamente 3.000 millones de pesos, con una cobertura de 1.800.000 jefes/as de hogar desocupados. (SIEMPRO, con base a datos del Ministerio de Trabajo y Seguridad Social). 
En el contexto mismo de la crisis en los últimos años de la década, fueron modelándose nuevos actores - aunque en muchos casos recuperando experiencias políticas previas -; nos referimos a los movimientos de trabajadores desocupados en sus distintas manifestaciones políticas, las redes de trueque (Hintze, 2003), las empresas recuperadas, ${ }^{10} \mathrm{y}$ las asambleas barriales. ${ }^{11}$

Con las diferencias a que cada uno de ellos nos enfrenta, se trata de propuestas de nuevas formas de organización colectiva y resignificación de la democracia que refieren a un desplazamiento de la macro representación a la micro construcción de voluntad política, a través de la jerarquización de la calidad de los procesos cara a cara, referidos a territorios concretos y con altos niveles de reflexividad (Hintze, 2003, p. 6). Un fenómeno a destacar es la existencia de experiencias que integran las formas de protesta con la gestión de distintas iniciativas o proyectos de producción o autoconsumo orientadas a lograr la independencia socioeconómica mejorando las condiciones de reproducción de las familias, así como reorientando programas de empleo en el desarrollo de emprendimientos (Ibid, p. 12).

La experiencia de los Consejos Consultivos Municipales muestra espacios diversos tanto en su conformación (los actores que participan), como en los alcances de la acción pública. La tipología elaborada a partir de un estudio nacional realizado hacia principios del 2003 (Siempro - Cenoc, 2003) muestra cinco configuraciones particulares:

$\mathrm{CC}$ formalizados pero con escasa incidencia en el ámbito local;

$\mathrm{CC}$ orientados a la función de auditoría;

$\mathrm{CC}$ promotores de proyectos comunitarios o socio productivos;

CC promotores de desarrollo local; $y$

$\mathrm{CC}$ sostenidos por la propia iniciativa de miembros de organizaciones de la sociedad civil.

10 Se trata de una cantidad creciente de emprendimientos que alcanzan desde la industria metalúrgica hasta el sector educativo, y dan lugar a la organización de cooperativas de trabajo o la propuesta de estatización bajo control obrero y son gestionadas por los ex empleados de establecimientos en convocatoria, quebrados o a punto de cerrar (Hintze, 2003, p. 9).

11 Un análisis de las alternativas políticas que estos nuevos actores sociales formulan ante tal crisis de representación puede encontrarse en Hintze, 2003, p. 9. 
El análisis de facilitadores y obstáculos de estos procesos nos hacen poner la atención en dos aspectos: por una parte, la capacidad de gobierno tanto de los actores gubernamentales como de la sociedad, como factor crítico para su desarrollo y pasaje a modelos superadores de lo asistencial; por la otra, la necesaria reflexión acerca de lo que se le puede pedir a los CC y lo que no. Esta parece ser una reflexión pendiente.

En suma, nos encontramos con una arena local compleja, diversa y heterogénea, modelada por las políticas, fragmentada, con actores con desigual capacidad de acción pública, de gestión y credibilidad.

Esbozada esta situación, la pregunta es cuál puede ser el papel de una estrategia nacional o provincial en el sentido de convertir a estos actores en sujetos de las políticas.

\section{La tarea estratégica: fortalecer los espacios de gestión pública participativa orientada hacia el desarrollo local}

El Plan Nacional de Desarrollo Local y Economía Social, del cual el "Manos a la Obra"12 es uno de sus componentes más importantes, reconoce la necesidad de que el Estado se constituya en articulador de las políticas a la vez que promueva la consolidación de los vínculos entre las organizaciones gubernamentales y de la sociedad civil ${ }^{13}$ en función de contribuir con el desarrollo de emprendimientos de la economía social orientados hacia el desarrollo local. En la perspectiva del Plan, economía social y desarrollo local son conceptos que se relacionan estrechamente.

El Plan supone un nuevo rol para los Consejos Consultivos que, trascendiendo la administración del Plan Jefes y Jefas de HD, se conviertan en espacios asociativos articuladores de políticas sociales y generadores de proyectos productivos. ${ }^{14}$ Esta iniciativa significa un cambio difícil y audaz en las reglas

$12<$ www.desarrollosocial.gov.ar $>$.

13 Aunque los proyectos para el componente "Manos a la Obra" pueden ser presentados por Municipios, Organizaciones de la Sociedad Civil o Consejos Consultivos, esta orientación se ve expresada en que se priorizarán aquellos proyectos avalados por Consejos Consultivos Provinciales y Municipales. Ver condiciones para presentación proyectos en sitio web: $<$ www.desarrollosocial.gov.ar $>$.

14 Los resultados de la experiencia de la Provincia de Buenos Aires deberían ser evaluados en este sentido dado que en sus objetivos iniciales se planteó convertir a los Consejos Consultivos en Consejos Económico Sociales Locales (CLES). 
de juego (expresado, entre otras en el replanteo de programas para integrarlos en ejes acotados de políticas, ${ }^{15}$ en la promoción de vínculos entre actores gubernamentales y de la sociedad) y también en las representaciones a las que se apela e insiste en fortalecer (la necesidad de fundar otra economía centrada en el trabajo, nuevas formas de construcción de legitimidad, otra forma de concebir la acción de gobierno, por señalar las más difundidas).

Desde la dinámica de los emprendimientos, las investigaciones sobre los emprendimientos sociales orientados hacia el desarrollo de la economía social o solidaria señalan al apoyo y seguimiento como factor crítico para su sostenibilidad. Esto llama la atención sobre la necesidad de apoyar el desarrollo de instancias subnacionales que puedan tener presencia cotidiana pero que sean promovidas, estimuladas, orientadas y fortalecidas por el nivel central, cuando sea necesario.

En este contexto y dadas estas orientaciones, la generación de espacios de gestión pública participativa para el desarrollo local es un desafío que requiere de una estrategia específica que la promueva, apoye y sostenga con la finalidad de recuperar la capacidad de intervención del poder público (en los distintos niveles) en tanto regulador de la vida social. Sin embargo, y según lo dicho más arriba, ni todos los entramados locales están en condiciones de avanzar en procesos de este tipo ni aquellos que han avanzado sostenidamente encuentran un camino exento de problemas.

Parte del balance realizado en distintas oportunidades permite insistir en esta línea. A pesar de las críticas y dificultades que encierran las experiencias de gestión de los Consejos Consultivos del Plan Jefas y Jefes de HD, las organizaciones sociales comunitarias siguen manifestando el interés institucional en participar de estos espacios multiactorales, valorizan la importancia de ser convocadas como sujetos de las políticas no sólo para acceder a recursos para resolver demandas, sino para participar en su diseño; aunque coinciden en que distan aún bastante de constituirse en espacios de gestión pública participativa. $^{16}$

15 Los programas existentes están integrados en tres ejes Producción Solidaria, Redes y Seguridad Alimentaria.

16 Conclusiones sobre el Seminario Nacional sobre Espacios Asociativos para la Gestión de las Políticas Sociales realizado por el CENOC el 24 y 25 de junio del 2003. 
Estas consideraciones nos permiten afirmar que una política que pretenda convocar y fortalecer espacios de gestión pública participativa debe tener en cuenta que no va a poder llevarse a cabo en todas las localidades y que en todas ellas tendrá que ser asumida como proceso de aprendizaje, no sólo de los actores que forman parte del espacio local, sino también de aquellos que actúan desde la nación o las provincias. La calidad de los proyectos y sus consecuencias en la sustentabilidad, el desarrollo de estrategias de comercialización y las posibilidades de estructuración de cadenas de valor entre emprendimientos sociales, depende directamente de la posibilidad de organizar este entramado que los promueva, apoye y sostenga.

Ahora bien, el desarrollo de una política en este sentido requiere de acciones de distinto tipo pero básicamente interpela a sus actores, en particular al Estado en sus distintos niveles y organizaciones, a poner en juego capacidades que no son exactamente idénticas a las desarrolladas en la década pasada. Para el Estado (en sus diferentes niveles y enfrentándose a conflictos de diversa naturaleza), implica recuperar la capacidad de impulsar procesos de negociación entre los distintos actores con el propósito de mejorar la calidad de vida de su población.

Desde las instancias centrales de gobierno, la necesidad de pensar este objetivo como objetivo de política requiere necesariamente del involucramiento de los distintos organismos (economía, trabajo, producción, infraestructura, salud, educación, entre otros) y no sólo a las áreas relacionadas con "lo social".

Las instancias provincials tienen un papel muy importante a desempeñar impulsando procesos de convocatoria y negociación entre actores, facilitando articulaciones regionales, generando servicios de apoyo, articulando con el sistema educativo y sanitario, y facilitando la participación de actores como las universidades, a fin de lograr la organización de un sistema de apoyo en capacitación, asistencia técnica y financiamiento.

Promovidas estas estrategias, los gobiernos locales se enfrentan a la tensión entre el mandato electoral y la participación de distintas organizaciones en el transcurso del periodo electivo; la operación sobre esta tensión es compleja en tanto la tarea consiste a la vez en desmontar las estructuras administrativas y corporativas armadas en función de la lógica electoral, y no sólo la estructura partidaria que es la que se nos aparece como la más evidente. Los 
distintos espacios de participación tienen la particularidad de tensionar la relación entre lo particular con lo general, de contraponer demandas específicas a necesidades de satisfacción de interés general; ${ }^{17}$ frente a estas tensiones se pone en juego un modo diferente de gobernar.

Es muy importante destacar que no nos estamos refiriendo a un nuevo proceso de transferencia de responsabilidades a las organizaciones sociales y menos aún de empoderamiento del tercer sector. Las iniciativas frente a la crisis, las apuestas de la sociedad y de los gobiernos están marcando que las fronteras entre el Estado y la sociedad no tiene límites precisos y dados, sino que son resultado de presiones y negociaciones.

Esta propuesta tampoco tiene como prerrequisito la complementariedad armónica; pensamos que esa articulación se resuelve - en algunos casos - por la vía del conflicto. Lo central de esta mirada es pensar que el Estado tiene una responsabilidad principal en garantizar un pacto político de inclusión y ese debe ser el objetivo que oriente a la gestión pública.

El desarrollo de una política en este sentido exige un cambio en el paradigma que orientó el desarrollo de capacidades institucionales en la década pasada; esto es, tenemos que pasar del desarrollo de capacidades para que la sociedad se haga cargo de lo que antes se hacía el Estado a fortalecer las capacidades de gobierno. ${ }^{18}$

Cuando hablamos de capacidades de gobierno nos referimos no solo a actores gubernamentales sino también a los actores de la sociedad (organizaciones de base y las ONGs). Nos estamos refiriendo tanto a las capacidades técnico administrativas como a las capacidades políticas, especialmente a la posibilidad de articular intereses en propuestas.

$\mathrm{Si}$, como sostuvimos más arriba, consideramos a estos procesos de desarrollo local como UNICOS y a las capacidades de gobierno como CRÍTICAS no existen "expertos" que - por fuera de la posibilidad de operar sobre este sistema de relaciones sociales y políticas - puedan hacer un aporte que resulte significativo y sostenible.

17 A estas tensiones hace alusión para el caso brasilero, S. Caccia Bava (2003).

18 Para el debate en relación al concepto de capacidad institucional y la necesidad de un enfoque relacional puede consultarse F. Repetto (2003). 
No se trata solo de poner en funcionamiento operaciones de corte técnico, frente a este desafío se trata de movilizar procesos sociales y políticos (con su componente de reglas de juego, prácticas y representaciones) que estén muy bien informados por contenidos rigurosos sobre los aspectos sociales, económicos y técnicos.

Por último, parte de la construcción de una identidad de estos espacios locales de gestión pasa por la posibilidad de definir políticas que trasciendan los programas desde los cuales se motorizan estos procesos y que involucren - en los procesos de desarrollo local - dimensiones más integrales de las políticas: salud, educación, infraestructura y ordenamiento urbano, por señalar las más relevantes. Además de la construcción de una identidad de gobiernos y sociedades comprometidas con el desarrollo local, esta aproximación facilita la sostenibilidad en el sentido de convertir al desarrollo en objetivo a la vez que condición contextual en cada una de estas dimensiones. ${ }^{19}$

El desarrollo local como objetivo remite a los contenidos, procesos institucionales intrasectoriales y la gestión estratégica en cada una de estas dimensiones; y también a las condiciones contextuales, en el sentido de necesidad de generar un proceso sinérgico, las expectativas y el contexto político institucional (sectorial y extrasectorial) para el desarrollo de politicas en cada una de esas dimensiones.

Una aproximación de este tipo favorece la promoción y sostenibilidad de los procesos orientados a la economía social y el desarrollo local a la vez que permite generar alianzas más amplias entre distintos sectores sociales desde la resolución de problemas.

\section{Referências}

AROCENA, J. Discutiendo lo local: las coordenadas del debate. Cuadernos CLAEH, $\mathrm{n}^{\mathrm{o}}$ 45/46, Montevideo, 1988.

BARREIRO CAVESTANY, F. Los agentes del desarrollo. Cuadernos del CLAEH, $\mathrm{n}^{\circ}$ 45/46, Montevideo, 1988.

CACCIA BAVA, S. Participación, representación y nuevas formas de diálogo público In: Pobreza Urbana y Desarrollo, Alianzas para el Desarrollo Local. Año 9, n 21, junio de 2003. Serie Programa FORTAL.

19 Para un análisis en esta línea relativo a educación ver Coraggio (2001). 
CENOC - Seminario Nacional sobre Espacios Asociativos para la Gestión de las Políticas Sociales, 24 y 25 de junio, 2003.

CHIARA, M y Di Virgilio, M. M. La gestión de programas sociales en la última década: un análisis en clave local. En: G. Badía y E. Pereyra, La Plata, UNGSEdiciones Al Margen (En Prensa). Aportes para entender la cuestión del gobierno en la Región Metropolitana de Buenos Aires.

CHIARA, M. y Di Virgilio, M. M. Las transformaciones de los lineamientos de las reformas de la política social en el nivel local: la gestión de programas de empleo y salud en un municipio del Gran Buenos Aires. En: VI Congreso Internacional del CLAD sobre la Reforma del Estado y de la Administración Pública. Argentina, 5 - 9 de noviembre de 2001.

CORAGGIO, J. C. El papel de la teoría en la promoción del desarrollo local (Hacia el desarrollo de una economía centrada en el trabajo). www.fronesis.org.ar, 2003.

CORAGGIO, J. L. Educación y Desarrollo Local. Conversación sostenida con integrantes de la Fundación SES (sustentabilidad, educación y solidaridad) el 19 de octubre del 2001, Buenos Aires (www.fronesis.org.ar).

DI VIRGILIO, M. M. Casa se busca. explorando las relaciones entre estrategias habitacionales, redes sociales y politicas sociales. IIGG-UBA / ICO-UNGS, 2001.

HINTZE, S. Democracia participativa y nuevos actores en la sociedad civil argentina. Mimeo, p. 9, 2003.

HINTZE, S. Comp. Trueque y Economía Solidaria. ICO/UNGS - PNUD, www.ungs.edu.ar, 2003.

JORDANA, J. El análisis de los policy networks: ¿una nueva perspectiva sobre la relación entre políticas pública y Estado? En: Gestión y Análisis de Políticas Públicas, $\mathrm{n}^{\circ}$ 3, mayo/agosto, 1995.

KOHAN, G. y FOURNIER, M. Estructura social y desigualdades socio-espaciales: El caso de la Región Metropolitana de Buenos Aires. Instituto del Conurbano, Universidad de General Sarmiento, 1999.

REPETTO, F. Capacidad Institucional: un camino para enfrentar los desafíos de la política social. En: Boletín Tecnología para la Organización Pública, 꾸 5, 2003.

SIEMPRO - CENOC - Consejo Nacional de Coordinación de Políticas Sociales. Evaluación de los niveles de participación de las organizaciones de la sociedad civil en los Consejos Consultivos y la capacidad de aprovechamiento de los recursos locales. Coord. D. Arroyo, 1999.

SUBIRATS, J. La puesta en práctica de las políticas públicas. En: Análisis de politicas públicas y eficacia, pp. 101-123, 1989. 